\title{
On real analytic orbifolds and Riemannian metrics
}

\author{
MARJA KANKAANRINTA
}

\begin{abstract}
We begin by showing that every real analytic orbifold has a real analytic Riemannian metric. It follows that every reduced real analytic orbifold can be expressed as a quotient of a real analytic manifold by a real analytic almost free action of a compact Lie group. We then extend a well-known result of Nomizu and Ozeki concerning Riemannian metrics on manifolds to the orbifold setting: Let $X$ be a smooth (real analytic) orbifold and let $\alpha$ be a smooth (real analytic) Riemannian metric on $X$. Then $X$ has a complete smooth (real analytic) Riemannian metric conformal to $\alpha$.
\end{abstract}

$57 \mathrm{R} 18$

\section{Introduction}

In this paper we consider Riemannian metrics on smooth, ie, $\mathbb{C}^{\infty}$, and real analytic orbifolds. As is well-known, a smooth Riemannian metric for any smooth orbifold can be constructed by using invariant Riemannian metrics on orbifold charts and gluing them together by a smooth partition of unity. Real analytic manifolds admit real analytic Riemannian metrics, since they can be real analytically embedded in Euclidean spaces. Neither of these two methods to construct Riemannian metrics work for real analytic orbifolds and a different approach is needed.

Recall that an orbifold is called reduced if the actions of the finite groups on orbifold charts are effective. We first study the frame bundle $\operatorname{Fr}(X)$ of a reduced $n$-dimensional real analytic orbifold $X$. The frame bundle $\operatorname{Fr}(X)$ is a real analytic manifold and the general linear group $\mathrm{GL}_{n}(\mathbb{R})$ acts properly and almost freely, ie, with finite isotropy subgroups, on $\operatorname{Fr}(X)$. Thus $\operatorname{Fr}(X)$ has a $\mathrm{GL}_{n}(\mathbb{R})$-invariant real analytic Riemannian metric (see Illman and the author [4, Theorem I]), which induces a real analytic Riemannian metric on $X$. We then show that every real analytic orbifold inherits a real analytic Riemannian metric from the corresponding reduced orbifold. Therefore we obtain the following.

Theorem 1.1 Let $X$ be a real analytic orbifold. Then $X$ has a real analytic Riemannian metric. 
Since, by Theorem 1.1, every real analytic orbifold has a real analytic Riemannian metric, we can construct the orthonormal frame bundle $\operatorname{OFr}(X)$ for every reduced real analytic orbifold. As in the smooth case (see Adem, Leida and Ruan [1, Theorem 1.23]), we prove the following.

Theorem 1.2 Let $X$ be a reduced $n$-dimensional real analytic orbifold. Then $X$ is real analytically diffeomorphic to the quotient orbifold $\mathrm{OFr}(X) / \mathrm{O}(n)$.

Notice that if $X$ is a reduced $n$-dimensional real analytic orbifold, then $\operatorname{OFr}(X)$ is a real analytic manifold with a real analytic, effective, almost free action of the orthogonal group $\mathrm{O}(n)$. Thus Theorem 1.2 implies the following.

Corollary 1.3 Let $X$ be a reduced $n$-dimensional real analytic orbifold. Then $X$ is real analytically diffeomorphic to a quotient orbifold $M / \mathrm{O}(n)$, where $M$ is a real analytic manifold and $\mathrm{O}(n)$ acts on $M$ real analytically, effectively and almost freely.

It follows that reduced real analytic orbifolds can be studied by using methods developed for studying real analytic almost free actions of compact Lie groups.

To prove Theorems 1.1 and 1.2, we use two kinds of comparisons. Firstly, we compare Riemannian metrics on a quotient orbifold $M / G$ to $G$-invariant Riemannian metrics on the $G$-manifold $M$ (Section 3). Secondly, we compare Riemannian metrics on an orbifold to those on the corresponding reduced orbifold (Section 4). We conclude the paper by applying these comparisons to prove a result concerning complete Riemannian metrics.

Theorem 1.4 Let $X$ be a smooth (resp. real analytic) orbifold. For any smooth (resp. real analytic) Riemannian metric $\alpha$ on $X$ there exists a complete smooth (resp. real analytic) Riemannian metric on $X$ which is conformal to $\alpha$.

The corresponding result for Riemannian metrics on smooth manifolds has been proved by Nomizu and Ozeki [8, Theorem 1]. The corresponding equivariant result, which also is used in the proof of Theorem 1.4, was proved by the author [5, Theorems 3.1 and 5.2].

Acknowledgements The author's research was supported by the visitors program of the Aalto University during the academic year 2012-2013. The author would like to thank the Department of Mathematics and Systems Analysis of the Aalto University for its hospitality during her stay. 


\section{Definitions}

We first recall the definition of an orbifold.

Definition 2.1 Let $X$ be a topological space and let $n \in \mathbb{N}$.

(1) An orbifold chart of $X$ is a triple $(\tilde{U}, G, \varphi)$, where $\tilde{U}$ is an open connected subset of $\mathbb{R}^{n}, G$ is a finite group acting on $\widetilde{U}$ and $\varphi: \tilde{U} \rightarrow X$ is a $G$-invariant map inducing a homeomorphism $U=\varphi(\tilde{U}) \cong \tilde{U} / G$. Let $\operatorname{ker}(G)$ be the subgroup of $G$ acting trivially on $\tilde{U}$.

(2) An embedding $(\lambda, \theta):(\tilde{U}, G, \varphi) \rightarrow(\tilde{V}, H, \psi)$ between two orbifold charts is an injective homomorphism $\theta: G \rightarrow H$ such that $\theta$ is an isomorphism from $\operatorname{ker}(G)$ to $\operatorname{ker}(H)$, and an equivariant embedding $\lambda: \tilde{U} \rightarrow \tilde{V}$ with $\psi \circ \lambda=\varphi$.

(3) An orbifold atlas on $X$ is a family $\mathcal{U}=\{(\tilde{U}, G, \varphi)\}$ of orbifold charts which cover $X$ and satisfy the following: For any two charts $(\tilde{U}, G, \varphi)$ and $(\tilde{V}, H, \psi)$ and a point $x \in \varphi(\widetilde{U}) \cap \psi(\tilde{V})$, there exist a chart $(\widetilde{W}, K, \mu)$ such that $x \in \mu(\widetilde{W})$ and embeddings $(\widetilde{W}, K, \mu) \rightarrow(\widetilde{U}, G, \varphi)$ and $(\widetilde{W}, K, \mu) \rightarrow(\widetilde{V}, H, \psi)$.

(4) An orbifold atlas $\mathcal{U}$ refines another orbifold atlas $\mathcal{V}$ if every chart in $\mathcal{U}$ can be embedded into some chart in $\mathcal{V}$. Two orbifold atlases are equivalent if they have a common refinement.

Definition 2.2 An $n$-dimensional orbifold is a paracompact Hausdorff space $X$ equipped with an equivalence class of $n$-dimensional orbifold atlases.

An orbifold is called smooth (resp. real analytic), if for every orbifold chart ( $\tilde{U}, G, \varphi), G$ acts smoothly (resp. real analytically) on $\tilde{U}$ and if each embedding $\lambda: \tilde{U} \rightarrow \tilde{V}$ is smooth (resp. real analytic).

Let $X$ be an orbifold, and let $x \in X$. Let $(\tilde{U}, G, \varphi)$ and $(\tilde{V}, H, \psi)$ be orbifold charts of $X$ such that $x \in \varphi(\tilde{U}) \cap \psi(\tilde{V})$. Let $\tilde{x} \in \tilde{U}$ and $\tilde{y} \in \tilde{V}$ be such that $\varphi(\tilde{x})=\psi(\tilde{y})=x$. We denote the isotropy subgroups at $\tilde{x}$ and $\tilde{y}$ by $G_{\tilde{x}}$ and $H_{\tilde{y}}$, respectively. Then $G_{\tilde{x}}$ and $H_{\tilde{y}}$ are isomorphic. Thus we can associate to every $x \in X$ a finite group, welldefined up to an isomorphism, and called the local group of $x$.

Orbifold maps are defined as follows.

Definition 2.3 Let $X$ and $Y$ be smooth (real analytic) orbifolds. We call a map $f: X \rightarrow Y$ a smooth (real analytic) orbifold map, if for every $x \in X$, there are charts $(\tilde{U}, G, \varphi)$ around $x$ and $(\tilde{V}, H, \psi)$ around $f(x)$, such that $f$ maps $U=\varphi(\tilde{U})$ into $V=\psi(\widetilde{V})$ and the restriction $f \mid U$ can be lifted to a smooth (real analytic) equivariant 
map $\tilde{f}: \tilde{U} \rightarrow \tilde{V}$. A smooth (real analytic) map $f: X \rightarrow Y$ is called a smooth (real analytic) diffeomorphism if $f$ is a bijection and if the inverse map $f^{-1}: Y \rightarrow X$ is smooth (real analytic).

Remark Let $X$ be an orbifold and let $F$ be any finite group. Replace every orbifold chart $(\tilde{U}, G, \varphi)$ of $X$ by the orbifold chart $(\tilde{U}, G \times F, \varphi)$, where $G \times F$ acts on $\tilde{U}$ via the projection $G \times F \rightarrow G$. Doing this for every orbifold chart of $X$ yields an orbifold $Y$. The identity maps $X \rightarrow Y$ and $Y \rightarrow X$ are orbifold maps, and they are smooth (real analytic) if $X$ and $Y$ are smooth (real analytic) orbifolds. This means that two orbifolds are not necessarily considered equivalent, even if they are diffeomorphic. However, if there is a diffeomorphism $f: X \rightarrow Y$, where both $X$ and $Y$ are reduced orbifolds, then $X$ and $Y$ have equivalent sheaf categories; see Moerdijk and Pronk [7, Proposition 2.1]. In particular, in this case the local groups of $x$ and $f(x)$ are isomorphic, for every $x \in X$.

Definition 2.4 A Riemannian metric $\alpha$ on an orbifold $X$ is given by a collection of Riemannian metrics $\alpha^{\widetilde{U}}$ on the $\tilde{U}$ of the orbifold charts $(\tilde{U}, G, \varphi)$ so that

(1) the group $G$ acts isometrically on $\tilde{U}$,

(2) the embeddings $\widetilde{W} \rightarrow \widetilde{U}$ and $\widetilde{W} \rightarrow \widetilde{V}$ of Definition 2.1(3) are isometries.

If $X$ is a smooth (real analytic) orbifold and if all the $\alpha^{\widetilde{U}}$ are smooth (real analytic), then $\alpha$ is a smooth (real analytic) Riemannian metric.

Let $X$ be a smooth orbifold, and let $\left(\tilde{U}_{i}, G_{i}, \varphi_{i}\right), i \in I$, be orbifold charts of $X$ such that $\left\{\varphi_{i}\left(\widetilde{U}_{i}\right)\right\}_{i \in I}$ is a locally finite cover of $X$. Then each $\tilde{U}_{i}$ has a smooth Riemannian metric $\alpha^{\widetilde{U}_{i}}$, and by averaging over $G_{i}$, we may assume that $\alpha^{\widetilde{U}_{i}}$ is $G_{i}$-invariant, ie, that $G_{i}$ acts isometrically on $\tilde{U}_{i}$. Gluing these Riemannian metrics together, by using a smooth partition of unity, gives a smooth Riemannian metric on $X$; see Moerdijk and Mrčun [6, Proposition 2.20]. All the orbifolds in [6] are assumed to be reduced. However, the proof of Proposition 2.20 also works in the general case.

We next recall the way to define distance on a connected Riemannian orbifold; for details and proofs, see Borzellino [3]. Assume a smooth (real analytic) orbifold $X$ is equipped with a smooth (real analytic) Riemannian metric $\alpha$. Let $\gamma:[0,1] \rightarrow X$ be an admissible curve [3, Definition 35]. The interval $[0,1]$ can be decomposed into finitely many subintervals $\left[t_{i}, t_{i+1}\right]$ such that $\gamma\left(\left[t_{i}, t_{i+1}\right]\right) \subset U_{i}=\varphi\left(\tilde{U}_{i}\right)$, for some chart $\left(\tilde{U}_{i}, G_{i}, \varphi_{i}\right)$ of $X$. Let $\gamma_{i}$ denote the restriction of $\gamma$ to $\left[t_{i}, t_{i+1}\right]$, and let $\tilde{\gamma}_{i}$ be a lift of $\gamma_{i}$, for every $i$. If $\tilde{\gamma}_{i}$ is piecewise differentiable, its length can be calculated by integrating. If $\tilde{\gamma}_{i}$ is merely continuous, then its length can be calculated by approximating it by 
piecewise differentiable curves. Every lift of $\gamma_{i}$ has the same length and the length of the lift does not depend on which chart of $X$ is being used. Thus the length $L_{\alpha}\left(\gamma_{i}\right)$ of $\gamma_{i}$ can be defined to be the length of $\tilde{\gamma}_{i}$. Then the length $L_{\alpha}(\gamma)$ of $\gamma$ equals the sum of the $L_{\alpha}\left(\gamma_{i}\right)$.

Every curve connecting two points on an orbifold can always be replaced by an admissible curve whose local lifts are at most as long as the ones of the original curve [3, Remark 39]. Thus the distance between any given points $x$ and $y$ of a connected orbifold $X$ can be defined to be

$$
d_{\alpha}(x, y)=\inf \left\{L_{\alpha}(\gamma) \mid \gamma \text { is an admissible curve joining } x \text { to } y\right\} .
$$

Then $X$ equipped with the metric $d_{\alpha}$ becomes a metric space. If $d_{\alpha}$ is a complete metric, then any two points on $X$ can be joined by a minimal geodesic realizing the distance $d_{\alpha}(x, y)$ [3, Theorem 40]. Moreover, $X$ is a locally compact length space. Thus it follows that $d_{\alpha}$ is a complete metric if and only if the metric balls in $X$ are relatively compact.

\section{Riemannian metric on a quotient orbifold}

Let $G$ be a Lie group and let $M$ be a smooth (real analytic) manifold. Assume $G$ acts on $M$ by a smooth (real analytic) almost free action. Assume also that the action is proper, ie, that the map

$$
G \times M \rightarrow M \times M, \quad(g, x) \mapsto(g x, x),
$$

is proper. It is well-known that the quotient $M / G$ is a smooth (real analytic) orbifold and that every smooth (real analytic) $G$-invariant Riemannian metric on $M$ induces a smooth (real analytic) Riemannian metric on $M / G$. We present a proof of this basic result here (Theorem 3.1), since we failed to find one in the literature.

The main idea is to use the differentiable slice theorem: Let $x \in M$ and let $G x$ denote the orbit of $x$. Let $G_{x}$ denote the isotropy subgroup of $G$ at $x$. A $G$-invariant neighbourhood of $x$ can be equipped with a smooth (real analytic) $G$-invariant Riemannian metric. Then there is a $G_{X}$-invariant smooth (real analytic) submanifold $\mathrm{N}_{x}$ of $M$ that contains $x$ and is $G_{x}$-equivariantly diffeomorphic to an open $G_{x}$-invariant neighbourhood of the origin in the normal space $\mathrm{T}_{x}(M) / \mathrm{T}_{x}(G x)$ to $G x$ at $x$. The manifold $\mathrm{N}_{x}$ is called a linear slice at $x$. It intersects the orbit $G x$ orthogonally, and it intersects every orbit it meets transversely but not necessarily orthogonally. The exponential map takes an open neighbourhood of the zero section of the normal bundle 
of $G x$ diffeomorphically to the neighbourhood $G \mathrm{~N}_{x}$ of $G x$ which can be identified with the twisted product $G \times_{G_{x}} \mathrm{~N}_{x}$. The map

$$
f: G \mathrm{~N}_{x} \cong G \times_{G_{x}} \mathrm{~N}_{x} \rightarrow G / G_{x} \cong G x, \quad g y \mapsto g x,
$$

is smooth (real analytic) and $G$-equivariant. The map $f$ is exactly the map that assigns to every point $z$ in $G \mathrm{~N}_{x}$ the unique nearest point $f(z)$ in $G x$. Thus, if $g \in G$ and $y \in \mathrm{N}_{x}$, then the distance from $g y$ to $G x$ equals $d(g y, g x)=d(y, x)$, where $d$ denotes the metric induced by the local Riemannian metric on the connected components of $G \mathrm{~N}_{x}$.

Let $x \in M$ and let $\mathrm{N}_{x}$ be a linear slice at $x$ constructed by using a local $G$-invariant Riemannian metric $\alpha_{1}$. There is a real analytic local cross section $\delta: U \rightarrow G$ of the map $G \rightarrow G / G_{X}, g \mapsto g G_{x}$, defined in some $G_{X}$-invariant neighbourhood $U$ of $e G_{x}$ in $G / G_{x}$ and having the property $\delta\left(e G_{x}\right)=e$. We may choose $\delta$ to be $G_{x}$-equivariant, ie, $\delta(h u)=h \delta(u) h^{-1}$, for every $h \in G_{X}$ and for every $u \in U$. Let $f_{0}: G \mathrm{~N}_{x} \rightarrow G / G_{x}, g y \mapsto g G_{x}$. The map $F: U \times \mathrm{N}_{x} \rightarrow V,(u, s) \mapsto \delta(u) s$, is a smooth (real analytic) diffeomorphism onto some neighbourhood $V$ of $\mathrm{N}_{x}$. The inverse of $F$ is given by $F^{-1}: V \rightarrow U \times \mathrm{N}_{x}, y \mapsto\left(f_{0}(y), \delta\left(f_{0}(y)\right)^{-1} y\right)$. Let then $y \in \mathrm{N}_{x}$ and let $\mathrm{N}_{y}^{\prime}$ be a linear slice at $y$ constructed by using a local $G$-invariant Riemannian metric $\alpha_{2}$. We may assume that $\mathrm{N}_{y}^{\prime} \subset V$. Let pr: $U \times \mathrm{N}_{x} \rightarrow \mathrm{N}_{x}$ be the projection, and let $\lambda=\operatorname{pr} \circ F^{-1} \mid: \mathrm{N}_{y}^{\prime} \rightarrow \mathrm{N}_{x}$. Then $\lambda$ is an equivariant embedding and it induces the identity map on the orbit space level.

It follows that the quotient $M / G$ is an orbifold with orbifold charts $\left(\mathrm{N}_{x}, G_{x}, \pi_{x}\right)$, where $x \in M$ and $\pi_{x}$ denotes the natural projection $\mathrm{N}_{x} \rightarrow \mathrm{N}_{x} / G_{x} \cong\left(G \mathrm{~N}_{x}\right) / G$. The $\mathrm{N}_{x}$ are defined by using local $G$-invariant Riemannian metrics.

Let us next consider a smooth (real analytic) $G$-invariant Riemannian metric $\alpha$ on $M$. For every $x \in M$, let $\mathrm{N}_{x}$ be a linear slice constructed by using $\alpha$. Then $\alpha$ induces a smooth (real analytic) $G_{x}$-invariant Riemannian metric $\alpha \mid \mathrm{N}_{x}$ on $\mathrm{N}_{x}$, for every $x$. (The inner product on $\mathrm{T}_{z} \mathrm{~N}_{x}, z \in \mathrm{N}_{x}$, is given by first projecting to $\mathrm{T}_{z} \mathrm{~N}_{z}$ and then composing with $\alpha$.) Thus $G_{x}$ acts isometrically on $\mathrm{N}_{x}$, for every $x \in M$. By construction, the embeddings $\mathrm{N}_{y} \rightarrow \mathrm{N}_{x}$ are isometries. Let then $\mathrm{N}_{x}^{\prime}$ be a linear slice at $x$ defined by using some local $G$-invariant Riemannian metric. The map $\lambda: \mathrm{N}_{x}^{\prime} \rightarrow \mathrm{N}_{x}$ induces a smooth (real analytic) $G_{x}$-invariant Riemannian metric $\lambda_{*}\left(\alpha \mid \mathrm{N}_{x}\right)$ on $\mathrm{N}_{x}^{\prime}$. Thus also $\lambda$ is an isometry. Consequently, the Riemannian metrics obtained on the linear slices satisfy the conditions of Definition 2.4. Therefore, $\alpha$ induces a smooth (real analytic) Riemannian metric on $M / G$. We have proved the following. 
Theorem 3.1 Let $G$ be a Lie group and let $M$ be a smooth (real analytic) manifold on which $G$ acts by a proper, smooth (real analytic) almost free action. Then the quotient $M / G$ is a smooth (real analytic) orbifold. Every smooth (real analytic) $G-$ invariant Riemannian metric $\alpha$ on $M$ induces a smooth (real analytic) Riemannian metric $\hat{\alpha}$ on $M / G$.

We leave it for the reader to verify the following observation.

Lemma 3.2 Let $G$ be a Lie group and let $M$ be a smooth (real analytic) manifold on which $G$ acts by a proper, smooth (real analytic) almost free action. Assume $M / G$ is connected. Let $M_{0}$ be a connected component of $M$, and let $H=\left\{g \in G \mid g M_{0}=M_{0}\right\}$. Then the following hold:

(1) $H$ is a closed subgroup of $G$, and it contains the connected component $G_{0}$ of the identity element of $G$;

(2) the quotient orbifolds $M / G$ and $M_{0} / H$ are canonically smoothly (real analytically) diffeomorphic;

(3) there is a one-to-one correspondence between smooth (real analytic) $G$-invariant Riemannian metrics on $M$ and smooth (real analytic) $H$-invariant Riemannian metrics on $M_{0}$;

(4) there is a one-to-one correspondence between smooth (real analytic) $G$-invariant maps $M \rightarrow \mathbb{R}$ and smooth (real analytic) $H$-invariant maps $M_{0} \rightarrow \mathbb{R}$.

Let $G, M$ and $\alpha$ be as in Theorem 3.1. Let $x \in M$ and let $\mathrm{N}_{x}$ be a linear slice at $x$, defined by using $\alpha$. Let $\delta: U \rightarrow G$ be a real analytic cross section of the map $G \rightarrow G / G_{x}, g \mapsto g G_{x}$, as before Theorem 3.1. Let $F: U \times \mathrm{N}_{x} \rightarrow V$, be the smooth (real analytic) diffeomorphism defined by using $\delta$, and let pr: $U \times \mathrm{N}_{x} \rightarrow \mathrm{N}_{x}$ denote the projection. Let $\gamma:[0,1] \rightarrow V \subset G \mathrm{~N}_{x}$ be a curve. The map pro $F^{-1}$ takes every point in $V$ to a point in the same orbit. Thus the curves $\operatorname{pr} \circ F^{-1} \circ \gamma$ and $\gamma$ induce the same curve $[0,1] \rightarrow M / G$. Assume there is $c \in(0,1)$ such that $\gamma(c)=x$. Let $\gamma_{0}$ be the geodesic segment connecting $\left(\mathrm{pr} \circ F^{-1} \circ \gamma\right)(0)$ to $x$ and let $\gamma_{1}$ be the geodesic segment connecting $x$ to $\left(\operatorname{pr} \circ F^{-1} \circ \gamma\right)(1)$. Then the two geodesic segments are contained in $\mathrm{N}_{x}$ and they intersect orthogonally the $G$-orbits they meet (see Alekseevsky, Kriegl, Losik and Michor [2], the proof of Proposition 3.1(2)). Let $\gamma^{*}$ denote the curve $\gamma_{0} \cup \gamma_{1}$. We obtain the following lemma.

Lemma 3.3 For every curve $\gamma:[0,1] \rightarrow V$ such that $\gamma(c)=x$, for some $c \in(0,1)$, there is a curve $\gamma^{*}:[0,1] \rightarrow \mathrm{N}_{x}$ having the following properties:

(1) $L_{\widehat{\alpha}}\left(\gamma^{*}\right)=L_{\alpha}\left(\gamma^{*}\right) \leq L_{\alpha}(\gamma)$;

(2) $\pi\left(\gamma^{*}(0)\right)=\pi(\gamma(0))$ and $\pi\left(\gamma^{*}(1)\right)=\pi(\gamma(1))$. 
We point out that for any curve $\gamma$ in $\mathrm{N}_{x}, L_{\hat{\alpha}}(\gamma)$ denotes the length of $\gamma$ calculated by using the Riemannian metric $\alpha \mid \mathrm{N}_{x}$ defined before Theorem 3.1, while $L_{\alpha}(\gamma)$ denotes the length of $\gamma$ calculated by using the $G_{x}$-invariant submanifold Riemannian metric $\alpha$ induces on $\mathrm{N}_{x}$. If $\gamma$ intersects orthogonally every orbit it meets, then the two lengths are the same.

Assume $M / G$ is connected. Let $M_{0}$ be a connected component of $M$, and let $H$ be the subgroup of $G$ consisting of the elements that map $M_{0}$ to itself, as in Lemma 3.2. Let $\alpha$ be a smooth (real analytic) $G$-invariant Riemannian metric on $M$. By restriction, we may consider $\alpha$ as an $H$-invariant Riemannian metric on $M_{0}$. Let $d_{\alpha}$ be the $H$-invariant metric induced on $M_{0}$ by $\alpha$. The metric $d_{\alpha}$ then induces a metric $\tilde{d}_{\alpha}$ on $M_{0} / H \cong M / G$, where

$$
\tilde{d}_{\alpha}(\pi(x), \pi(y))=\inf \left\{d_{\alpha}(x, h y) \mid h \in H\right\} .
$$

Let $d_{\widehat{\alpha}}$ be the metric that the Riemannian metric $\hat{\alpha}$ induces on $M / G$. We will use Lemma 3.3 to prove the following result.

Theorem 3.4 Let $M, G, \alpha$ and $\hat{\alpha}$ be as in Theorem 3.1. Assume $M / G$ is connected. Then $\tilde{d}_{\alpha}=d_{\hat{\alpha}}$.

Proof By Lemma 3.2, we may without loss of generality assume that $M$ is connected. Let $x, y \in M$. We will show $\widetilde{d}_{\alpha}(\pi(x), \pi(y))=d_{\widehat{\alpha}}(\pi(x), \pi(y))$. Let $\gamma:[0,1] \rightarrow M / G$ be a curve such that $\gamma(0)=\pi(x)$ and $\gamma(1)=\pi(y)$. We may assume that $\gamma$ is admissible. Let $\tilde{\gamma}:[0,1] \rightarrow M$ be a lift of $\gamma$. Decompose the interval $[0,1]$ into finitely many subintervals $\left[t_{i}, t_{i+1}\right], 1 \leq i \leq m$, such that $\tilde{\gamma}\left(\left[t_{i}, t_{i+1}\right]\right)$ is contained in a small neighbourhood $V_{i} \cong U_{i} \times \mathrm{N}_{x_{i}}$ of $\mathrm{N}_{x_{i}}$, as before Lemma 3.3, where $\mathrm{N}_{x_{i}}$ is a linear slice at $x_{i} \in \tilde{\gamma}\left(\left[t_{i}, t_{i+1}\right]\right)$. We may assume that $x_{1}=\tilde{\gamma}(0)$ and $x_{m}=\tilde{\gamma}(1)$. For every $1<i<m$, let $c_{i} \in\left(t_{i}, t_{i+1}\right)$ be such that $\tilde{\gamma}\left(c_{i}\right)=x_{i}$. Let $\tilde{\gamma}_{i}$ denote the restriction of $\tilde{\gamma}$ to $\left[t_{i}, t_{i+1}\right]$, for every $i$. By Lemma 3.3, we may replace every curve $\tilde{\gamma}_{i}$ by a curve $\tilde{\gamma}_{i}^{*}:\left[t_{i}, t_{i+1}\right] \rightarrow \mathrm{N}_{x_{i}}$ having the properties that $\pi\left(\tilde{\gamma}_{i}^{*}\left(t_{i}\right)\right)=\pi\left(\tilde{\gamma}_{i}\left(t_{i}\right)\right)$, $\pi\left(\tilde{\gamma}_{i}^{*}\left(t_{i+1}\right)\right)=\pi\left(\tilde{\gamma}_{i}\left(t_{i+1}\right)\right)$ and

$$
L_{\widehat{\alpha}}\left(\widetilde{\gamma}_{i}^{*}\right)=L_{\alpha}\left(\widetilde{\gamma}_{i}^{*}\right) \leq L_{\alpha}\left(\tilde{\gamma}_{i}\right) .
$$

We next show that the $\tilde{\gamma}_{i}^{*}$ can be chosen in such a way that they define a curve $\tilde{\gamma}^{*}:[0,1] \rightarrow M$, where $\pi\left(\tilde{\gamma}^{*}(0)\right)=\pi(x)$ and $\pi\left(\tilde{\gamma}^{*}(1)\right)=\pi(y)$. For example, $\pi\left(\tilde{\gamma}_{1}^{*}\left(t_{2}\right)\right)=\pi\left(\tilde{\gamma}_{2}^{*}\left(t_{2}\right)\right), \tilde{\gamma}_{1}^{*}\left(t_{2}\right) \in \mathrm{N}_{x_{1}}$ and $\tilde{\gamma}_{2}^{*}\left(t_{2}\right)=g \tilde{\gamma}_{1}^{*}\left(t_{2}\right)$, for some $g \in G$. Thus, if $\tilde{\gamma}_{1}^{*}\left(t_{2}\right) \neq \tilde{\gamma}_{2}^{*}\left(t_{2}\right)$, we can replace $\tilde{\gamma}_{2}^{*}$ by $g^{-1} \circ \tilde{\gamma}_{2}^{*}$. Continuing like this, we can replace every $\tilde{\gamma}_{i}^{*}$, if necessary, in such a way that we obtain a curve $\tilde{\gamma}^{*}:[0,1] \rightarrow M$. The 
curve $\tilde{\gamma}^{*}$ induces a curve $\gamma^{*}:[0,1] \rightarrow M / G$ with $\gamma^{*}(0)=\pi(x)$ and $\gamma^{*}(1)=\pi(y)$. It follows from the way $\gamma^{*}$ was constructed that

$$
L_{\widehat{\alpha}}\left(\gamma^{*}\right)=L_{\alpha}\left(\tilde{\gamma}^{*}\right) \leq L_{\alpha}(\tilde{\gamma}) .
$$

Since $\gamma$ was an arbitrary path from $\pi(x)$ to $\pi(y)$, it follows that

$$
d_{\widehat{\alpha}}(\pi(x), \pi(y)) \leq \tilde{d}_{\alpha}(\pi(x), \pi(y)) .
$$

Let then $z \in M$ and let $\mathrm{N}_{z}$ be a linear slice at $z$. Let $\mu:[0,1] \rightarrow \mathrm{N}_{z}$ be a curve. We may assume that $\mu$ is simple, starts at $z$ and intersects each orbit at most once. If $\mu([0,1])$ is orthogonal to every orbit it meets, then $L_{\alpha}(\mu)=L_{\hat{\alpha}}(\mu)$. If $\mu([0,1])$ is not orthogonal to every orbit it meets, then we may replace $\mu$ by a curve $\mu^{*}:[0,1] \rightarrow G \mathrm{~N}_{z}$ with $\mu^{*}(t) \in G \mu(t)$, for every $t \in[0,1]$, such that $\mu^{*}([0,1])$ is orthogonal to every orbit it meets. Then

$$
L_{\widehat{\alpha}}(\mu)=L_{\alpha}\left(\mu^{*}\right) \geq \tilde{d}_{\alpha}(\pi(\mu(0)), \pi(\mu(1))) .
$$

Replacing local lifts of any path from $\pi(x)$ to $\pi(y)$ in this manner and gluing them at the endpoints shows that

$$
\tilde{d}_{\alpha}(\pi(x), \pi(y)) \leq d_{\widehat{\alpha}}(\pi(x), \pi(y)) .
$$

According to [5, Lemma 2.4], the metric $\tilde{d}_{\alpha}$ on $M / G \cong M_{0} / H$ is complete if and only if the $H$-invariant metric $d_{\alpha}$ on $M_{0}$ is complete. Since, by Theorem 3.4, $\tilde{d}_{\alpha}=d_{\widehat{\alpha}}$, it follows that $d_{\hat{\alpha}}$ is complete if and only if $d_{\alpha}$ is complete. We conclude with the following corollary.

Corollary 3.5 Let $G$ be a Lie group and let $M$ be a smooth (real analytic) manifold on which $G$ acts by a proper, smooth (real analytic) almost free action. Let $\alpha$ be a $G$-invariant smooth (real analytic) Riemannian metric on $M$ and let $\hat{\alpha}$ be the smooth (real analytic) Riemannian metric that $\alpha$ induces on $M / G$. Then $\hat{\alpha}$ is complete if and only if $\alpha$ is complete.

\section{Comparing Riemannian metrics on $X$ and $X_{\text {red }}$}

Let $X$ be a smooth (real analytic) orbifold. Assume $X$ is not reduced. Replacing every orbifold chart $(\tilde{U}, G, \varphi)$ by a chart $(\tilde{U}, G / \operatorname{ker}(G), \varphi)$ yields a smooth (real analytic) reduced orbifold $X_{\text {red }}$. The orbifolds $X$ and $X_{\text {red }}$ are identical as topological spaces and the identity map $X \rightarrow X_{\text {red }}$ is an orbifold map. Let $(\tilde{U}, G, \varphi)$ be an orbifold chart of $X$. Then a Riemannian metric on $\tilde{U}$ is invariant under the action of $G$ if and only if it is invariant under the action of $G / \operatorname{ker}(G)$. The following proposition follows immediately from Definition 2.4. 
Proposition 4.1 There is a one-to-one correspondence between Riemannian metrics on $X$ and Riemannian metrics on $X_{\text {red }}$. A Riemannian metric $\alpha$ on $X$ is smooth (real analytic) if and only if the corresponding Riemannian metric $\alpha_{\text {red }}$ on $X_{\text {red }}$ is smooth (real analytic).

Remark Assume $X$ is connected. Let $d_{\alpha}$ and $d_{\alpha_{\text {red }}}$ be the metrics induced on $X$ by $\alpha$ and on $X_{\text {red }}$ by $\alpha_{\text {red }}$, respectively. If we just consider $X$ and $X_{\text {red }}$ as topological spaces, ie, if we identify $X_{\text {red }}$ with $X$, then both $d_{\alpha}$ and $d_{\alpha_{\text {red }}}$ are metrics on $X$ and $d_{\alpha}=d_{\alpha_{\text {red }}}$. In particular, this implies that $\alpha$ is complete if and only if $\alpha_{\text {red }}$ is complete.

\section{Real analytic Riemannian metric}

In this section we show that every real analytic orbifold has a real analytic Riemannian metric. In order to do that, we first need to construct the frame bundle $\operatorname{Fr}(X)$ of a reduced real analytic orbifold $X$. The construction is similar to that in the smooth case. For details, see [6, Pages 42-43].

Recall that, for an $n$-dimensional real analytic manifold, the frame bundle $\operatorname{Fr}(M)$ is a real analytic fibre bundle over $M$, the fibre of $x \in M$ is the manifold of all ordered bases of the tangent space $\mathrm{T}_{x}(M)$. The frame bundle $\operatorname{Fr}(M)$ admits a canonical right action of the general linear group $\mathrm{GL}_{n}(\mathbb{R})$ which makes it a principal $\mathrm{GL}_{n}(\mathbb{R})$-bundle over $M$.

For a reduced $n$-dimensional real analytic orbifold $X$, we first form the frame bundles $\operatorname{Fr}\left(\tilde{U}_{i}\right)$ corresponding to orbifold charts $\left(\tilde{U}_{i}, G_{i}, \varphi_{i}\right)$. The action of $G_{i}$ on $\tilde{U}_{i}$ induces a left action on $\operatorname{Fr}\left(\tilde{U}_{i}\right)$,

$$
G_{i} \times \operatorname{Fr}\left(\tilde{U}_{i}\right) \rightarrow \operatorname{Fr}\left(\tilde{U}_{i}\right), \quad\left(g,\left(x, B_{x}\right)\right) \mapsto\left(g x,(d g)_{x}\left(B_{x}\right)\right) .
$$

Since $G_{i}$ acts effectively on $\tilde{U}_{i}$, it follows that the action of $G_{i}$ on $\operatorname{Fr}\left(\tilde{U}_{i}\right)$ is free. The group $\mathrm{GL}_{n}(\mathbb{R})$ acts on $\operatorname{Fr}\left(\tilde{U}_{i}\right)$ from the right and the action commutes with the action of $G_{i}$. Thus $\operatorname{Fr}\left(\tilde{U}_{i}\right) / G_{i}$ is a real analytic manifold on which $\mathrm{GL}_{n}(\mathbb{R})$ acts real analytically. In fact, we can consider $\operatorname{Fr}\left(\tilde{U}_{i}\right) / G_{i}$ as a twisted product $\tilde{U}_{i} \times{ }_{G_{i}} \mathrm{GL}_{n}(\mathbb{R})$. It now follows from $[4$, Lemma 0.1$]$, that $\mathrm{GL}_{n}(\mathbb{R})$ acts properly on $\operatorname{Fr}\left(\tilde{U}_{i}\right) / G_{i}$.

Assume $A \in \mathrm{GL}_{n}(\mathbb{R})$ and $[x, I] A=[x, I]$. Then $(x, A)=\left(g x,(d g)_{x}\right)$, for some $g \in G_{i}$. Thus $g \in\left(G_{i}\right)_{x}$ and $A=(d g)_{x}$. It follows that the isotropy subgroups of the $\mathrm{GL}_{n}(\mathbb{R})$-action are finite, ie, $\mathrm{GL}_{n}(\mathbb{R})$ acts almost freely on $\operatorname{Fr}\left(\tilde{U}_{i}\right) / G_{i}$.

The frame bundle $\operatorname{Fr}(X)$ of $X$ can be constructed by gluing together the quotients $\operatorname{Fr}\left(\tilde{U}_{i}\right) / G_{i}$. This is done by using the gluing maps induced by the embeddings $\lambda_{i j}: \widetilde{U}_{i} \rightarrow \widetilde{U}_{j}$ between orbifold charts. We obtain the following. 
Theorem 5.1 Let $X$ be a reduced $n$-dimensional real analytic orbifold. Then the frame bundle $\operatorname{Fr}(X)$ of $X$ is a real analytic manifold on which $\mathrm{GL}_{n}(\mathbb{R})$ acts by a proper, real analytic, effective, almost free action. The orbifolds $X$ and $\operatorname{Fr}(X) / \mathrm{GL}_{n}(\mathbb{R})$ are real analytically diffeomorphic.

We are now ready to prove Theorems 1.1 and 1.2.

Proof of Theorem 1.1 Let us first assume that $X$ is a reduced $n$-dimensional real analytic orbifold. By Theorem 5.1, $X \cong \operatorname{Fr}(X) / \mathrm{GL}_{n}(\mathbb{R})$. Since $\mathrm{GL}_{n}(\mathbb{R})$ acts properly and real analytically on $\operatorname{Fr}(X)$, it follows from [4, Theorem I], that $\operatorname{Fr}(X)$ has a real analytic $\mathrm{GL}_{n}(\mathbb{R})$-invariant Riemannian metric $\alpha$. But then, by Theorem 3.1, $\alpha$ induces a real analytic Riemannian metric on $X$.

Let then $X$ be any real analytic orbifold, and let $X_{\text {red }}$ be the corresponding reduced orbifold. By the first part of the proof, we know that $X_{\text {red }}$ has a real analytic Riemannian metric. It now follows from Proposition 4.1, that also $X$ has a real analytic Riemannian metric.

Proof of Theorem 1.2 Let $X$ be a reduced $n$-dimensional real analytic orbifold. Since $X$ has a real analytic Riemannian metric, by Theorem 1.1, we can construct the orthonormal frame bundle $\operatorname{OFr}(X)$ of $X$ (denoted by $\operatorname{Fr}(X)$ in [1]), exactly as in the smooth case, see [1, Pages 11-12]. The proof is now similar to the proof of the smooth case [1, Theorem 1.23].

The following result is well-known; see Stanhope and Uribe [9, Proposition 2.1] for the smooth case. The proof of the real analytic case is similar.

Proposition 5.2 Let $X$ be a reduced $n$-dimensional smooth (real analytic) orbifold and let $\operatorname{OFr}(X)$ be the orthonormal frame bundle of $X$. Let $\beta$ be a smooth (real analytic) Riemannian metric on $\operatorname{OFr}(X) / \mathrm{O}(n)$. Then there is an $\mathrm{O}(n)$-invariant smooth (real analytic) Riemannian metric $\alpha$ on $\operatorname{OFr}(X)$ such that $\beta$ equals the Riemannian metric $\hat{\alpha}$ induced on $\operatorname{OFr}(X) / \mathrm{O}(n)$ by $\alpha$.

Theorem 1.2, Corollary 3.5 and Propositions 5.2 and 4.1 and the remark after it imply the following correspondence.

Corollary 5.3 Let $X$ be an $n$-dimensional smooth (real analytic) orbifold, and let $X_{\text {red }}$ be the reduced orbifold corresponding to $X$. Then every smooth (real analytic) Riemannian metric on $X$ is induced by an $\mathrm{O}(n)$-invariant smooth (real analytic) Riemannian metric on $\operatorname{OFr}\left(X_{\text {red }}\right)$. Conversely, any $\mathrm{O}(n)$-invariant smooth (real analytic) Riemannian metric on $\operatorname{OFr}\left(X_{\text {red }}\right)$ induces a smooth (real analytic) Riemannian metric on $X$. A Riemannian metric on $X$ is complete if and only if it is induced by a complete $\mathrm{O}(n)$-invariant Riemannian metric on $\operatorname{OFr}\left(X_{\text {red }}\right)$. 


\section{Complete Riemannian metric}

Recall that two smooth (real analytic) Riemannian metrics $\alpha_{1}$ and $\alpha_{2}$ on a smooth (real analytic) orbifold $X$ are called conformal, if there exists a smooth (real analytic) orbifold map $\omega: X \rightarrow \mathbb{R}$ such that $\omega(x)>0$ for every $x \in X$ and $\alpha_{1}=\omega \alpha_{2}$.

Proof of Theorem 1.4 Let id: $X \rightarrow X_{\text {red }}$ be the identity map. By [1, Theorem 1.23] and Theorem 1.2, we have that there is a smooth (real analytic) diffeomorphism $f: X_{\text {red }} \rightarrow \operatorname{OFr}\left(X_{\text {red }}\right) / \mathrm{O}(n)$. Let $\pi: \operatorname{OFr}\left(X_{\text {red }}\right) \rightarrow \operatorname{OFr}\left(X_{\text {red }}\right) / \mathrm{O}(n)$ denote the natural projection. Let $\alpha$ be a smooth (real analytic) Riemannian metric on $X$, and let $\alpha_{\text {red }}$ be the corresponding Riemannian metric on $X_{\text {red }}$. The diffeomorphism $f$ induces a smooth (real analytic) Riemannian metric $f^{*} \alpha_{\text {red }}$ on $\operatorname{OFr}\left(X_{\text {red }}\right) / \mathrm{O}(n)$. By Proposition 5.2, there is an $\mathrm{O}(n)$-invariant smooth (real analytic) Riemannian metric $\beta$ on $\operatorname{OFr}\left(X_{\text {red }}\right)$ such that the Riemannian metric $\hat{\beta}$ induced on $\operatorname{OFr}\left(X_{\text {red }}\right) / \mathrm{O}(n)$ by $\beta$ equals $f^{*} \alpha_{\text {red }}$. By [5, Theorems 3.1 and 5.2], there is an $\mathrm{O}(n)$-invariant smooth (real analytic) map $\omega: \operatorname{OFr}\left(X_{\text {red }}\right) \rightarrow \mathbb{R}$ such that the Riemannian metric $\omega^{2} \beta$ on $\operatorname{OFr}\left(X_{\text {red }}\right)$ is complete. Let $\bar{\omega}: \operatorname{OFr}\left(X_{\text {red }}\right) / \mathrm{O}(n) \rightarrow \mathbb{R}$ denote the map induced by $\omega$. Then $\left(\bar{\omega}^{2} \circ f \circ \mathrm{id}\right) \alpha$ is a complete smooth (real analytic) Riemannian metric on $X$ conformal to $\alpha$.

A Riemannian metric $\alpha$ on a connected orbifold $X$ is called bounded if $X$ is bounded with respect to the metric induced by $\alpha$. The following result concerning bounded Riemannian metrics was originally proved by Nomizu and Ozeki in the manifold setting [8, Theorem 2].

Theorem 6.1 Let $X$ be a connected smooth (real analytic) orbifold and let $\alpha$ be a smooth (real analytic) Riemannian metric on $X$. Then there is a bounded smooth (real analytic) Riemannian metric on $X$ which is conformal to $\alpha$.

Proof We use the same notation as in the proof of Theorem 1.4. By Theorem 1.4, we may assume that $\alpha$ is complete. Let $x_{0}$ be an arbitrary point in $\operatorname{OFr}\left(X_{\text {red }}\right)$ and let $\operatorname{OFr}\left(X_{\text {red }}\right)_{0}$ denote the connected component of $\operatorname{OFr}\left(X_{\text {red }}\right)$ containing $x_{0}$. Let $H=\left\{h \in \mathrm{O}(n) \mid h\left(\mathrm{OFr}\left(X_{\text {red }}\right)_{0}\right)=\mathrm{OFr}\left(X_{\text {red }}\right)_{0}\right\}$. (In fact, $H=\mathrm{O}(n)$, or $H=\mathrm{SO}(n)$.) Let $\beta$ be the $\mathrm{O}(n)$-invariant smooth (real analytic) Riemannian metric on $\operatorname{OFr}\left(X_{\text {red }}\right)$ such that the Riemannian metric $\hat{\beta}$ induced on $\operatorname{OFr}\left(X_{\text {red }}\right) / \mathrm{O}(n)$ by $\beta$ equals $f^{*} \alpha_{\text {red }}$, and let $\beta_{0}$ denote the restriction of $\beta$ to $\operatorname{OFr}\left(X_{\text {red }}\right)_{0}$. Let $d_{\beta_{0}}$ denote the $H$-invariant metric $\beta_{0}$ induces on $\operatorname{OFr}\left(X_{\text {red }}\right)_{0}$. Let

$$
r_{0}: \operatorname{OFr}\left(X_{\text {red }}\right)_{0} \rightarrow \mathbb{R}, \quad x \mapsto \max \left\{d_{\beta_{0}}\left(h x_{0}, x\right) \mid h \in H\right\} .
$$


Then $r_{0}$ is a continuous $H$-invariant map and $r_{0}(x) \geq d_{\beta_{0}}\left(x_{0}, x\right)$ for all $x \in \operatorname{OFr}\left(X_{\text {red }}\right)_{0}$. By [5, Lemmas 2.3 and 5.1], there is an $H$-invariant smooth (real analytic) map $r: \operatorname{OFr}\left(X_{\text {red }}\right)_{0} \rightarrow \mathbb{R}$ such that $r(x)>r_{0}(x)$, for all $x \in \operatorname{OFr}\left(X_{\text {red }}\right)_{0}$. The Riemannian metric $e^{-2 r} \beta_{0}$ on $\operatorname{OFr}\left(X_{\text {red }}\right)_{0}$ is $H$-invariant and, by the proof of Theorem 2 in [8], it is bounded. Let $\bar{r}: \operatorname{OFr}\left(X_{\text {red }}\right) / \mathrm{O}(n) \cong \mathrm{OFr}\left(X_{\text {red }}\right)_{0} / H \rightarrow \mathbb{R}$ denote the map induced

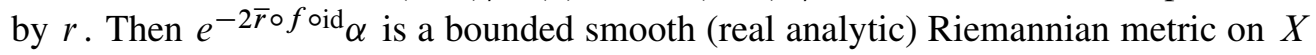
and it is conformal to $\alpha$.

Assume every Riemannian metric on $X$ is complete. According to Theorem 6.1, $X$ has a bounded complete Riemannian metric. Thus it follows that $X$ must be compact.

\section{References}

[1] A Adem, J Leida, Y Ruan, Orbifolds and stringy topology, Cambridge Tracts in Mathematics 171, Cambridge Univ. Press (2007) MR2359514

[2] D Alekseevsky, A Kriegl, M Losik, P W Michor, The Riemannian geometry of orbit spaces-the metric, geodesics, and integrable systems, Publ. Math. Debrecen 62 (2003) 247-276 MR2008095 Dedicated to Professor Lajos Tamássy on the occasion of his 80th birthday

[3] J E Borzellino, Riemannian geometry of orbifolds, $\mathrm{PhD}$ Thesis, University of California, Los Angeles (1992) Available at http://search.proquest.com/docview/ 304009974

[4] S Illman, M Kankaanrinta, Three basic results for real analytic proper G-manifolds, Math. Ann. 316 (2000) 169-183 MR1735083

[5] M Kankaanrinta, Some basic results concerning G-invariant Riemannian metrics, J. Lie Theory 18 (2008) 243-251 MR2413962

[6] I Moerdijk, J Mrčun, Introduction to foliations and Lie groupoids, Cambridge Studies in Advanced Mathematics 91, Cambridge Univ. Press (2003) MR2012261

[7] I Moerdijk, D A Pronk, Orbifolds, sheaves and groupoids, K-Theory 12 (1997) 3-21 MR1466622

[8] K Nomizu, H Ozeki, The existence of complete Riemannian metrics, Proc. Amer. Math. Soc. 12 (1961) 889-891 MR0133785

[9] E Stanhope, A Uribe, The spectral function of a Riemannian orbifold, Ann. Global Anal. Geom. 40 (2011) 47-65 MR2795449

Department of Mathematics, University of Virginia PO Box 400137, Charlottesville, VA 22903, USA

mk5aq@virginia.edu

Received: 3 December 2012 